\title{
Screen-detected ductal carcinoma in situ found on stereotactic vacuum-assisted biopsy of suspicious microcalcifications without mass: radiological-histological correlation
}

\author{
Bartlomiej Szynglarewicz¹, Piotr Kasprzak², Przemyslaw Biecek³ , Agnieszka Halon4, \\ Rafal Matkowski ${ }^{1,5}$ \\ ${ }^{1}$ Breast Unit, Department of Surgical Oncology, Lower Silesia Oncology Centre, Wroclaw, Poland \\ ${ }^{2}$ Department of Breast Imaging, Lower Silesia Oncology Centre, Wroclaw, Poland \\ ${ }^{3}$ Faculty of Mathematics, Informatics and Mechanics, University of Warsaw, Warsaw, Poland \\ ${ }^{4}$ Department of Pathomorphology and Oncological Cytology, Wroclaw Medical University, Poland \\ ${ }^{5}$ Department of Oncology, Wroclaw Medical University, Poland
}

Radiol Oncol 2016; 50(2): 145-152.

Received 30 November 2015

Accepted 26 January 2016

Correspondence to: Bartlomiej Szynglarewicz, M.D., Lower Silesian Oncology Centre, Plac Hirszfelda 12, 53-413 Wroclaw, Poland. Phone: +48 071368 9333; Fax: +48 071799 8600. E-mail: szynglarewicz.b@dco.com.pl

Disclosure: No potential conflicts of interest were disclosed.

Background. Commonly identified on screening mammography breast microcalcifications are the predominant manifestation of ductal carcinoma in situ (DCIS). The aim of this study was to investigate the association between clinico-radiological features and histological findings in patients with screen-detected DCIS.

Patients and methods. Consecutive 127 patients with pure DCIS found on stereotactic vacuum-assisted biopsy of screen-detected suspicious microcalcifications without mass entered the study. Patient age, type and distribution of microcalcifications, DCIS nuclear grade (NG) and the presence of comedonecrosis were investigated. Association between parameters was statistically analysed with $\mathrm{P}<0.05$ as a significance level.

Results. Powdery microcalcifications were most often clustered while regional were most common of casting-type $(P<0.001)$. High, intermediate and low NG of DCIS was significantly related to casting-type, crushed stone-like and powdery microcalcifications, respectively $(P<0.01)$. Low and intermediate NG DCIS were the most common in clustered and grouped microcalcifications while high NG DCIS was the most often when regional distribution was observed $(P<0.05)$. Comedonecrosis was significantly more common in high NG DCIS $(P<0.01)$. The association between comedonecrosis and type of microcalcifications was not significant, but with their distribution was close to the significance level $(P=0.07)$. Patient age was not significantly related to imaging or histological findings.

Conclusions. The association between pattern of mammographic microcalcifications and histological findings related to more aggressive disease can be helpful in optimal surgery planning in patients with screen-detected DCIS, regarding the extent of breast intervention and consideration of synchronous sentinel node biopsy.

Key words: breast cancer screening; mammographic microcalcifications; ductal carcinoma in situ

\section{Introduction}

The development of imaging techniques and the widespread adoption of screening programs resulted in dramatically increased incidence of ductal carcinoma in situ (DCIS), which currently accounts for about $20-25 \%$ of newly diagnosed breast cancer cases. ${ }^{1}$ The most common clinical presentation of DCIS are mammographically visible microcalcifications. Although being present in about $30 \%$ of all breast cancers and in approximately $55 \%$ of nonpalpable breast malignancies, they are responsible 
for the detection of $85-95 \%$ of cases of DCIS by screening mammography. ${ }^{2,3}$

The microcalcifications associated with the development of DCIS arise in the lumen of the terminal ducts, by calcium production on the secretion material or on the zones of necrosis. ${ }^{4}$ They only indirectly attest to the cell proliferation of the carcinoma, that will then progress in the ducts in an anterograde direction toward the nipple or in a retrograde direction, within the lobule. ${ }^{5}$ The microcalcifications are commonly discontinuous, which may indicate multifocality. However, since the true multifocal DCIS is currently believed to be a relatively rare condition, they most often correspond to a single lesion extending to several ducts by contiguity. ${ }^{4-7}$ With the introduction of stereotactic minimal-invasive biopsy (core-needle or vacuumassisted) it has become possible to obtain a preoperative diagnosis of suspicious breast microcalcifications not visible on ultrasound. As most DCIS lesions are nonalpable and some are more extensive that suspected on the mammogram, evaluation of imaging-pathologic correlation by a multidisciplinary team is essential in the assessment of patients to determine their eligibility for breast conserving surgery as well as to achieve in these cases a complete excision with negative margins and good cosmetic outcomes. ${ }^{8}$

The aim of this study was to investigate the clinical and histological features, and to evaluate the association among these findings in screen-detected DCIS found on stereotactic vacuum-assisted biopsy of suspicious microcalcifications.

\section{Patients and methods}

\section{Screening program}

Nation-wide and population-based breast cancer screening program is targeting women aged 50-69, with exclusion of females undergoing treatment or being followed-up due to breast cancer. Twoview mammography (cranio-caudal and oblique) is used as a standard screening test. High-quality analogue (screen-film) or full field digital mammography (FFDM) are both allowed in screening program in Poland. Routine round length of the program is two years. All women with radiological findings categorised as suspicious of malignancy (BIRADS 4) or highly suggested for cancer (BIRADS 5) are referred to further assessment and invasive investigations. In patients with masses ultrasound-guided core-needle or vacuum-assisted biopsy is carried out, whereas in patients with oth- er lesions, not well seen in ultrasound, a stereotactic biopsy under digital mammography guidance is performed.

\section{Patients}

A cohort of 127 consecutive non-symptomatic patients with screen-detected DCIS diagnosed in years 2009-2014 was enrolled. All of them fulfilled the study entering criteria: BIRADS category 4 or 5 microcalcifications without mass or architectural distortion, pure DCIS found on histological examination, lack of invasion or microinvasion $(\leq 1 \mathrm{~mm}$ in the longest diameter), absence of any other breast malignancy or border-line lesion. Median (mean, range) patient age was 60 years (59.6, 50-69). All the patients underwent the same type of minimalinvasive biopsy under the stereotactic guidance. Study was approved by the Independent Ethics Committee (UMED KB-376) and the Institutional Board (NDOK/668). Patients signed the informed consent.

\section{Biopsy}

In each case an informed consent to undergo biopsy was obtained. All the procedures were performed by one breast-dedicated radiologist (PK), in the same breast care unit, according to the same standardised protocol to assure quality control. Five specimens was the minimal number of tissue cores. Each biopsy was done under the local anaesthesia using $10 \mathrm{~cm}^{3}$ of $1 \%$ lidocaine in two-step approach: $5 \mathrm{~cm}^{3}$ superficially and $5 \mathrm{~cm}^{3}$ deeply. Biopsies were completed under digital mammography guidance using a designated prone table unit (Mammotest Plus / S, Fisher Imaging, Denver, USA) with 10$G$ needle (EnCore Breast Biopsy System, SenoRx Inc., Irvine, CA or EnCore Enspire Breast Biopsy System, C.R. Bard Inc., Tempe, AZ).

\section{Pathology}

All hematoxylin and eosin stained slides of formalin-fixed and paraffin-embedded tissue blocks were assessed by board-certified pathologists experienced in breast cancer. In all the cases pathological examination reported pure DCIS without invasive or microinvasive component. In cases with any doubt about the origin E-cadherin immunochemistry was used to exclude a pleomorphic type of lobular carcinoma in situ. Evaluation of DCIS histology was performed according to the well-defined and widely accepted criteria. 


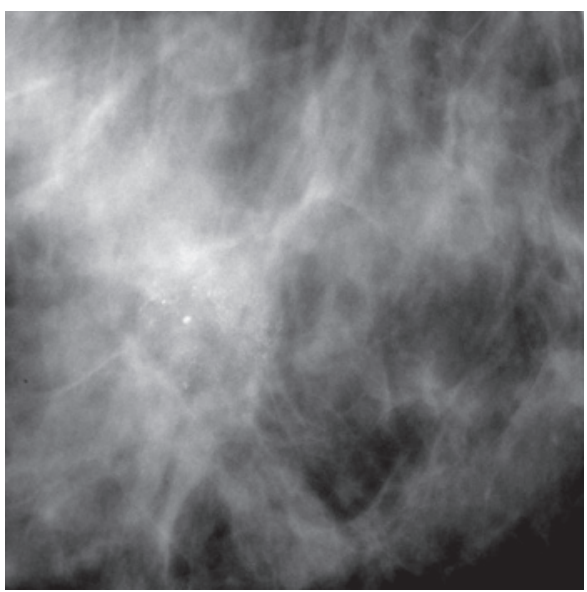

FIGURE 1. Powdery microcalcifications (cotton ball-like, indistinct, amorphous).

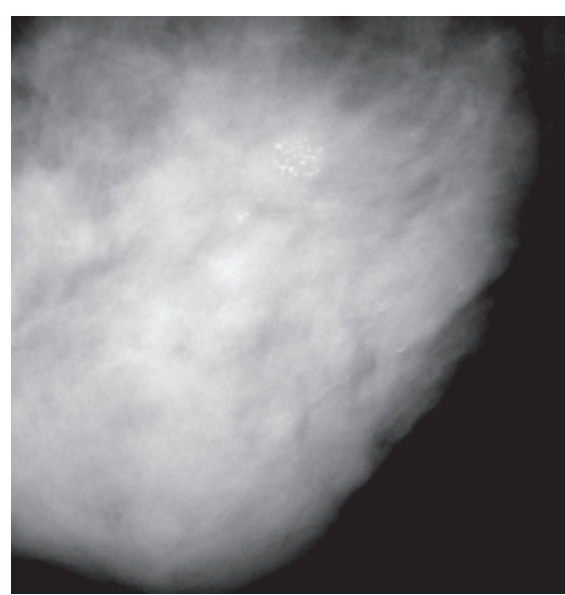

FIGURE 2. Crushed stone-like microcalcifications (pleomorphic).

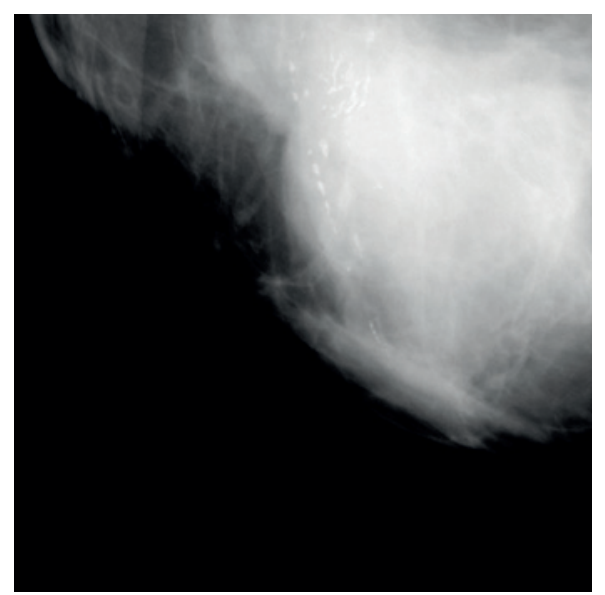

FIGURE 3. Casting-type microcalcifications (linear, branching).
Comedonecrosis was determined as present if the central areas of necrosis with ghost outlines of cells and cellular debris were found. Grading system was expressed by the assessment of nuclear grade (NG) categorising DCIS as low, intermediate, and high grade, based on the recommendations of the College of American Pathologists. ${ }^{9,10}$ Finally, all the slides were reviewed and re-evaluated by study supervising pathologist $(\mathrm{AH})$ to confirm the original diagnosis of DCIS and its histological features as well as the absence of invasion or microinvasion.

\section{Statistical analysis}

Data were collected in a prospective manner and entered a computer database. Statistical analysis was performed by professional statistician (PB). Following features were investigated: patient age, DCIS nuclear grade and the presence of comedonecrosis (as described above) as well as distribution pattern and morphology of microcalcifications. With regard to distribution pattern microcalcifications were classified as clustered $(<1 \mathrm{~cm})$, grouped $(1-2 \mathrm{~cm})$, and regional $(>2 \mathrm{~cm})$. Morphological type was categorised according to Tabar classification (Figure 1-3) as powdery (cotton ball-like: indistinct, amorphous), crushed stone-like (pleomorphic), and casting-type (linear, branching). ${ }^{11}$ Mammographic appearance was assessed before biopsy (without knowledge of the histopathological diagnosis) by two board-certified radiologists with special expertise in breast imaging (double reading) and then reviewed and re-evaluated by study supervising radiologist (PK) using analogue microfocus magnification techniques in orthogo-
TABLE 1. Baseline characteristics

\begin{tabular}{lc}
\hline Features & $\mathbf{n}(\%)$ \\
\hline Patient age & \\
50-60 & $67(53)$ \\
61-69 & $60(47)$ \\
Microcalcifications type & \\
Powdery & $19(15)$ \\
Crushed stone-like & $81(64)$ \\
Casting-type & $27(21)$ \\
Microcalcifications distribution & \\
Clustered & $85(67)$ \\
Grouped & $31(24)$ \\
Regional & $11(9)$ \\
Nuclear grade (NG) & \\
NG 1 (Low) & $71(56)$ \\
NG 2 (Intermediate) & $38(30)$ \\
NG 3 (High) & $18(14)$ \\
Comedonecrosis & \\
Absent & $58(46)$ \\
Present & $69(54)$ \\
\hline
\end{tabular}

nal planes or, in cases of FFDM, on-screen magnification. Baseline characteristics are presented in Table 1. Association between investigated variables was analysed using Pearson's chi-square test. $P$ values less than 0.05 were considered statistically significant.

\section{Results}

\section{Microcalcifications type - distribution}

$84 \%(\mathrm{n}=16)$ of powdery microcalcifications were clustered, $16 \%$ (3) were grouped, while none had regional distribution. Considering crushed stonelike and casting-type morphology 75\% (61) and $30 \%$ (8) of microcalcifications were clustered, $20 \%$ (16) and $44 \%$ (12) were grouped, $5 \%$ (4) and 26\% 
TABLE 2. Association between microcalcifications distribution and type

\begin{tabular}{lcccc}
\hline \multirow{2}{*}{ Features } & \multicolumn{3}{c}{ Microcalcifications distribution, $\mathrm{n}(\%)$} \\
\cline { 2 - 5 } & Clustered & Grouped & Regional \\
\hline Microcalcifications type, $\mathrm{n}[\%]$ & $16(19)[84]$ & $3(10)[16]$ & $0(-)[-]$ & {$[100 \%]$} \\
Powdery & $61(72)[75]$ & $16(51)[20]$ & $4(36)[5]$ & {$[100 \%]$} \\
Crushed stone-like & $8(9)[30]$ & $12(39)[44]$ & $7(64)[26]$ & {$[100 \%]$} \\
Casting type & $(100 \%)$ & $(100 \%)$ & $(100 \%)$ & $P<0.001$ \\
\hline
\end{tabular}

TABLE 3. Association between nuclear grade and microcalcifications

\begin{tabular}{|c|c|c|c|c|}
\hline \multirow{2}{*}{ Features } & \multicolumn{3}{|c|}{ Nuclear grade, n (\%) } & \\
\hline & I (Low) & II (Intermediate) & III (High) & \\
\hline \multicolumn{5}{|c|}{ Microcalcifications Type, n [\%] } \\
\hline Powdery & $14(20)[74]$ & $3(8)[16]$ & $2(11)[10]$ & [100\%] \\
\hline Crushed stone-like & $47(66)[58]$ & 27 (71) [33] & 7 (39) [9] & [100\%] \\
\hline \multirow[t]{2}{*}{ Casting type } & $10(14)[37]$ & $8(21)[30]$ & $9(50)$ [33] & {$[100 \%]$} \\
\hline & (100\%) & (100\%) & (100\%) & $P<0.01$ \\
\hline \multicolumn{5}{|l|}{ Distribution, n [\%] } \\
\hline Clustered & $54(76)[64]$ & $23(60)[27]$ & 8 (44) [9] & [100\%] \\
\hline Grouped & $14(20)[45]$ & 12 (32) [39] & $5(28)[16]$ & {$[100 \%]$} \\
\hline \multirow[t]{2}{*}{ Regional } & 3 (4) [27] & 3 (8) [27] & $5(28)[46]$ & [100\%] \\
\hline & (100\%) & (100\%) & (100\%) & $P<0.05$ \\
\hline
\end{tabular}

(7) had regional pattern, respectively. With regard to clustered and grouped microcalcifications 19\% (16) and 10\% (3) were powdery, 72\% (61) and 51\% (16) were crushed stone-like, while $9 \%(8)$ and 39\% (12) were casting-type, respectively. None of regional microcalcifications had powdery morphology, 36\% (4) were crushed stone-like, and 64\% (7) were casting-type. To sum up, powdery microcalcifications were most often clustered, never regional. Similarly, just one-fourth of crushed stonelike microcalcifications were grouped or regional. Clustered microcalcifications were most rarely of casting-type while regional were most commonly. The association between morphology and distribution of microcalcifications was of very high statistical significance $(\mathrm{t} 1 \mathrm{X}$-squared $=25.281, \mathrm{df}=4, \mathrm{P}=$ 4.416e-05) (Table 2).

\section{Microcalcifications type - nuclear grade}

All over $74 \%$ (14) of powdery microcalcifications revealed DCIS with low NG, 16\% (3) intermediate, and $10 \%$ (2) high NG. Crushed stone-like microc- alcifications were related to low NG in 58\% (47), intermediate in 33\% (27), and high in 9\% (7). In contrast, 33\% (9) of casting-type microcalcifications had high NG DCIS, 30\% (8) intermediate, while $37 \%$ (10) had low NG DCIS. 50\% (9) of high NG DCIS were presenting as casting-type microcalcifications, 39\% (7) as crushed stone-like, and 11\% (2) as powdery. $21 \%$ (8) of DCIS with intermediate NG was found in casting-type microcalcifications, 71\% (27) in crushed stone-like, and 8\% (3) in powdery. With regard to low NG DCIS, $14 \%$ (10) was detected in casting-type microcalcifications, while $66 \%$ (47) and 20\% (14) in crushed stone-like and powdery, respectively. In summary, high NG DCIS was most commonly related to casting-type microcalcifications while low NG DCIS most rarely. DCIS with intermediate NG was most often found in crushed stone-like microcalcifications. Just onefourth of powdery microcalcifications revealed DCIS with NG other than low. The association between morphology of microcalcifications and NG of DCIS was statistically significant ( $\mathrm{t} 1 \mathrm{X}$-squared = 13.363, $\mathrm{df}=4, \mathrm{P}=0.009632$ ) (Table 3). 


\section{Microcalcifications type - comedonecrosis}

Comedonecrosis was found in $47 \%$ (9) of powdery microcalcifications, $57 \%$ (46) of crushed stone-like, and in 52\% (14) of casting-type. DCIS with comedonecrosis was presenting as powdery in 13\% (10), crushed stone-like in $67 \%$ (46), and casting-type microcalcifications in $20 \%$ (14) of cases. Considering DCIS without comedonecrosis, powdery microcalcifications were detected in 17\% (10) of patients while crushed stone-like and casting-type in $60 \%$ (35) and 23\% (13), respectively.

\section{Microcalcifications type - patient age}

$58 \%$ (11) of patients with powdery microcalcifications were $50-60$ years old, whereas $54 \%$ (44) and $44 \%$ (12) of those with crushed stone-like and casting-type, respectively (Table 4). Patients aged 50-60 years had powdery microcalcifications in 16\% (11), crushed stone-like in $66 \%$ (44), and casting-type in $18 \%$ (12). Among older patients (61-69) powdery microcalcifications were present in $13 \%(8)$, crushed stone-like in $62 \%$ (37), and casting-type in $25 \%$ (15) of cases. Neither patient age nor the presence of comedonecrosis was significantly related to morphology of microcalcifications ( $\mathrm{t} 1 \mathrm{X}$-squared $=$ 1.0293, $\mathrm{df}=2, \mathrm{P}=0.5977 ; \mathrm{t} 1 \mathrm{X}$-squared $=0.63551, \mathrm{df}$ $=2, \mathrm{P}=0.7278$; respectively).

\section{Microcalcifications distribution - nuclear grade}

In $64 \%$ (54) of clustered microcalcifications low NG DCIS was found, whereas intermediate and high NG DCIS in 27\% (23) and 9\% (8), respectively. Grouped microcalcifications revealed low, intermediate and high NG DCIS in 45\% (14), 39\% (12), and $16 \%(5)$ of cases while regional microcalcifications in $27 \%$ (3), $27 \%$ (3), and $46 \%$ (5), respectively. $76 \%(54)$ and $60 \%$ (23) of DCIS with low and intermediate NG were presenting as clustered microcalcifications, $20 \%(14)$ and $32 \%(12)$ as grouped, and $4 \%$ (3) and $8 \%$ (3) as regional, respectively. Among patients with high NG DCIS $44 \%$ (8) had clustered, while $28 \%$ (5) each had grouped and regional microcalcifications. Summarising, low NG DCIS was the most common in clustered microcalcifications and very rare in regional, where high NG DCIS was the most often, being found in nearly half of cases. The association between distribution of microcalcifications and NG of DCIS was statis-
TABLE 4. Association between comedonecrosis and microcalcifications

\begin{tabular}{lccc}
\hline \multirow{2}{*}{ Features } & \multicolumn{2}{c}{ Comedonecrosis, $\mathbf{n}(\%)$} & \\
\cline { 2 - 3 } & Absent & Present & \\
\hline Microcalcifications Type, $\mathrm{n} \mathrm{[ \% ]}$ & $10(17)[53]$ & $9(13)[47]$ & {$[100 \%]$} \\
Powdery & $35(60)[43]$ & $46(67)[57]$ & {$[100 \%]$} \\
Crushed stone-like & $13(23)[48]$ & $14(20)[52]$ & {$[100 \%]$} \\
Casting type & $(100 \%)$ & $(100 \%)$ & $P>0.5$ \\
& & & \\
Distribution, $\mathrm{n}[\%]$ & $44(76)[52]$ & $41(59)[48]$ & {$[100 \%]$} \\
Clustered & $12(21)[39]$ & $19(28)[61]$ & {$[100 \%]$} \\
Grouped & $2(3)[18]$ & $9(13)[82]$ & {$[100 \%]$} \\
Regional & $(100 \%)(100 \%)$ & & P 0.07 \\
\hline
\end{tabular}

tically significant $(\mathrm{t} 1 \mathrm{X}$-squared $=13.233, \mathrm{df}=4$, $\mathrm{P}=0.01019)($ Table 3$)$.

\section{Microcalcifications distribution - comedonecrosis}

In $48 \%$ (41) of patients with clustered, $61 \%$ (19) with grouped and $82 \%(9)$ with regional microcalcifications DCIS with comedonecrosis was found. Among patients with non-comedo DCIS 76\% (44) had clustered, 21\% (12) had grouped, and 3\% (2) had regional microcalcifications. 59\% (41) of DCIS with comedonecrosis was presenting as clustered microcalcifications, while $28 \%$ (19) and $13 \%$ (9) as grouped and regional. In summary, three-fourths of patients without comedonecrosis had clustered microcalcifications while in the vast majority of patients with regional microcalcifications DCIS with comedonecrosis was diagnosed. The association between distribution of microcalcifications and the presence of comedonecrosis was close but did not reach the statistical significance $(\mathrm{t} 1 \mathrm{X}$-squared $=$ 5.2275, $\mathrm{df}=2, \mathrm{P}=0.07326$ ) (Table 4).

\section{Microcalcifications distribution - patient age}

All over 58\% (49) of patients with clustered, 39\% (12) with grouped, and 55\% (6) with regional microcalcifications were $50-60$ years old. Considering these younger patients (50-60), 73\% (49), 18\% (12), and $9 \%(6)$ had clustered, grouped and regional microcalcifications, respectively. Among older ones (61-69) clustered microcalcifications were found in $60 \%$ (36) while grouped and regional in 32\% (19) and $8 \%(5)$, respectively. Patient age was not sig- 
TABLE 5. Association between nuclear grade and comedonecrosis

\begin{tabular}{|c|c|c|c|c|}
\hline \multirow{2}{*}{ Features } & \multicolumn{3}{|c|}{ Nuclear grade, $n(\%)$} & \\
\hline & I (Low) & II (Intermediate) & III (High) & \\
\hline \multicolumn{5}{|c|}{ Comedonecrosis, n [\%] } \\
\hline \multirow[t]{2}{*}{ Present } & 34 (48) [49] & $18(47)[26]$ & $17(94)[25]$ & [100\%] \\
\hline & (100\%) & $(100 \%)$ & $(100 \%)$ & $P<0.01$ \\
\hline
\end{tabular}

TABLE 6. Statistical significance of dependency (chi-square test, P value)

\begin{tabular}{|c|c|c|c|c|c|}
\hline Features & Patient age & $\begin{array}{c}\text { Microcalcifications } \\
\text { type }\end{array}$ & $\begin{array}{l}\text { Microcalcifications } \\
\text { distribution }\end{array}$ & Nuclear grade & Comedonecrosis \\
\hline Patient age & - & 0.5977 & 0.1936 & 0.9098 & 1 \\
\hline Microcalcifications type & 0.5977 & - & $<0.001$ & $<0.01$ & 0.7278 \\
\hline $\begin{array}{l}\text { Microcalcifications } \\
\text { distribution }\end{array}$ & 0.1936 & $<0.001$ & - & $<0.05$ & 0.0733 \\
\hline Nuclear grade & 0.9098 & $<0.01$ & $<0.05$ & - & $<0.01$ \\
\hline
\end{tabular}

nificantly related to the distribution of microcalcifications ( $\mathrm{t} 1 \mathrm{X}$-squared $=3.2839, \mathrm{df}=2, \mathrm{P}=0.1936)$.

\section{Nuclear grade - comedonecrosis}

In $94 \%$ (17) of high NG DCIS the comedonecrosis was present, whereas in $47 \%$ (18) and $48 \%$ (34) of intermediate and low NG, respectively. Among patients without comedonecrosis 2\% (1) had high NG, while 34\% (20) and 64\% (37) had intermediate and low NG DCIS, respectively. In the group with comedonecrosis high, intermediate, and low NG DCIS were diagnosed in 25\% (17), 26\% (18), and $49 \%$ (34) of cases, respectively. In summary, almost all the patients with high NG DCIS had comedonecrosis while just less than half of others. In almost all the patients without comedonecrosis DCIS of low or intemediate NG was found. The association between NG and the presence of comedonecrosis was statistically significant $(\mathrm{t} 1 \mathrm{X}$-squared $=13.604$, $\mathrm{df}=2, \mathrm{P}=0.001112$ ) (Table 5).

\section{Nuclear grade - patient age}

Younger patients (50-60) had low, intermediate, and high NG DCIS in 57\% (38), 28\% (19), and 15\% (10), while older women (61-69) in 55\% (33), 32\% (19), and 13\% (8), respectively. In cases of high, intermediate, and low NG DCIS 56\% (10), 50\% (19), and 54\% (38) of patients were in younger age. Patient age was not significantly related to NG of DCIS (t1 X-squared $=0.18908, \mathrm{df}=2, \mathrm{P}=0.9098$ ).

\section{Comedonecrosis - patient age}

Comedonecrosis was present in 54\% (36) of younger patients and in 55\% (33) of those aged 61-69 years. When comedonecrosis was absent 53\% (31) of patients were 50-60 years old. Similarly, among patients with comedonecrosis $52 \%$ (36) was in younger group. The association was not significant (t1 X-squared =0, df =1, P=1).

Significance level of correlation between investigated variables is presented in Table 6.

\section{Discussion}

Surgery, sometimes followed by radiotherapy in cases with breast conservation, remains the treatment of choice in DCIS patients. Since it is highly favourable disease, there is no difference in mortality rate regardless of which treatment is chosen. ${ }^{12}$ On the other hand, the extent of surgical intervention and the need of postoperative radiotherapy depend on some well-defined factors known to be important in predicting of local recurrence. The most significant and independent variables are quantified by the University of California/ Van Nuys Prognostic Index (USC/VNPI), which is widely used in clinical practice. USC/VNPI is a numerical algorithm combining the following prognostic factors: age at diagnosis (older age is better), tumour size (smaller size is better), surgical margin width (wider margin is better), NG 
(lower grade is better), and the presence or absence of comedonecrosis (no necrosis is better). Each of the four predictors (NG and comedonecrosis both determine pathologic classification) is scored 1 (the most favourable), 2, or 3 (the least favourable), and then added together to give an overall score, ranging from a low of 4 (least likely to recur) to a high of 12 (most likely to recur). ${ }^{12}$

However, not all these variables are available before operation (e.g. microscopic lesion diameter, surgical margin width). Moreover, some of features available before surgery can be underestimated in specimens from minimal-invasive biopsy (e.g. low/intermediate nuclear grade, absence of comedonecrosis) and eventually upgraded in the final examination of postoperative specimen. It would be helpful in surgical treatment planning if the histological characteristics could be predicted from the mammogram, particularly from the type and distribution of microcalcifications, which are the most common imaging presentation of DCIS.

There are conflicting reports on whether the histological features of DCIS can be estimated by the pattern of microcalcifications found on mammography. Dinkel et al. found that linear branching microcalcifications tended to be associated with higher pathological grading. However, correlation was poor and not statistically significant. ${ }^{13}$ Also in a series of Slanetz and co-workers (75 cases, 62 with calcifications alone) histological grade of DCIS could not be accurately determined prospectively based on the mammographic appearance of microcalcifications. ${ }^{14}$ In contrast, in a study of Holland and Hendriks well-differentiated DCIS was most commonly associated with multiple clusters of fine granular microcalcifications while poorly differentiated DCIS usually appeared on the mammogram as either linear branching or as coarse granular microcalcifications. ${ }^{15}$ It corresponds to our observation that low NG DCIS is usually found in clustered microcalcifications of crushed stone-like or powdery type whereas high NG DCIS is most common in casting type microcalcifications with regional distribution.

Results of more recent studies support these findings. In the large dataset consisting of 1783 DCIS (Sloane Project) casting-type microcalcifications were more frequently seen in the higher grade of DCIS, occurring in 58\% of high grade while in $26 \%$ of low grade cases. Moreover, casting-type microcalcifications were increasingly common with increasing lesion size. ${ }^{16}$ These associations were of high statistical significance $(\mathrm{P}<0.001)$. De Roos et al. reported significant association between linear microcalcifications and high grade $(\mathrm{P}<0.001)$ as well as between fine granular type and low grade of DCIS $(\mathrm{P}<0.05) .{ }^{8}$ Barreau et al. studied a large cohort of 909 cases and found that granular or linear branching type and a number of microcalcifications higher than 20 were correlated with high grade of DCIS and the presence of comedonecrosis. ${ }^{17}$ Evans et al. noticed that when the comedonecrosis was present following features were seen more commonly: abnormal mammogram (95\% vs. $7 \%, \mathrm{P}<$ $0.001)$, mammogram with calcifications (96\% vs. $61 \%, \mathrm{P}<0.001)$, calcifications with a ductal distribution $(80 \%$ vs. $45 \%, \mathrm{P}<0.005)$, and rod-shaped calcifications $(83 \%$ vs. $45 \%, \mathrm{P}<0.001)$. In contrast, DCIS without comedonecrosis was associated with mammogram without calcifications (39\% vs. $4 \%$, P $<0.001)$ and predominantly punctate calcifications (36\% vs. $13 \%, \mathrm{P}<0.05) .{ }^{18}$ In a series of Stomper and Connolly predominantly linear calcifications were present in $47 \%$ of DCIS with comedonecrosis compared to $18 \%$ of DCIS without comedonecrosis (P $=0.01)$ while the predominantly granular calcifications in $53 \%$ and $82 \%(\mathrm{P}=0.01)$, respectively. ${ }^{19}$

Mammogram with microcalcifications is the most common imaging appearance of DCIS. Nevertheless, in some cases calcifications are not present. Interesting issue is to compare histological characteristics of calcified and non-calcified DCIS. As mentioned above, Evans and colleagues found that non-calcified DCIS was less commonly associated with comedonecrosis. ${ }^{18}$ Tang et al. reported that comedo DCIS had higher frequency of histologically seen calcifications in the ducts, however, when compared to other types it was easier to detect on ultrasound with not significant differences on mammography and MRI. ${ }^{20}$ Slanetz et al. noticed that DCIS presenting on mammography as only a mass was usually well-differentiated. ${ }^{14}$ In contrast, Cho et al. observed that $59 \%$ of non-calcified DCIS lesions were high grade or comedonecrosis type. ${ }^{21}$ Supporting this finding, in numerous recent studies the presence of accompanying mass was related to the increased risk of invasive ductal component, which can reflect more aggressive behaviour of DCIS. ${ }^{22-25}$ In the series of Rauch et al. DCIS more frequently visible on sonography was ER-negative type, which also tended to be larger, was more likely to be high grade ( $93 \%$ vs. $44 \%, \mathrm{P}<0.0001$ ) and associated with comedonecrosis ( $64 \%$ vs. $29 \%$, $\mathrm{P}<0.0001){ }^{26}$

Considering screen-detected DCIS, the presence of calcifications seems to be related to less favourable histology and features associated with more aggressive behaviour. In the analysis of 217 DCIS 
cases in 212 asymptomatic patients by Mun et al. high nuclear grade $(\mathrm{P}<0.05)$, comedonecrosis $(\mathrm{P}<$ $0.001)$, and the presence of HER2/neu oncogene $(\mathrm{P}<$ 0.001 ) were more common in the calcified lesions. ${ }^{27}$ Similarly, Kim et al. noticed that calcified DCIS was significantly more often HER2-positive than ERpositive or triple-negative. Histopathologically, HER2-positive DCIS and triple-negative DCIS were more commonly associated with high nuclear grade and comedonecrosis when compared to ERpositive DCIS. ${ }^{28}$

In the era of screening programmes, advanced diagnostic tools, image-guided minimal-invasive biopsies and oncoplastic surgery a very close cooperation in multidisciplinary team is essential for the optimum management of breast cancer patients. The correlation between pattern of mammographic microcalcifications and histological features related to more aggressive disease can be helpful in optimal surgery planning in patients with screen-detected DCIS, with regard to the extent of breast intervention and the consideration of synchronous sentinel node biopsy.

\section{Acknowledgements}

Authors would like to thank Ewa Kowalska for her excellent assistance in data collection and management.

\section{References}

1. Ernster VL, Ballard-Barbash R, Barlow WE, Zheng Y, Weaver DL, Cutter G, et al. Detection of ductal carcinoma in situ in women undergoing screening mammography. J Natl Cancer Inst 2002; 94: 1546-54.

2. Gajdos C, Tartter PI, Bleiweiss IJ, Hermann G, de Csepel J, Estabrook A, et al. Mammographic appearance of nonpalpable breast cancer reflects pathologic characteristics. Ann Surg 2002; 235: 246-51.

3. de Roos MA, van der Vegt B, de Vries J, Wesseling J, de Bock GH. Pathological and biological differences between screen-detected and interval ductal carcinoma in situ of the breast. Ann Surg Oncol 2007; 14: 2097-104.

4. Holland R, Hendriks JH, Vebeek AL, Mruvanac M, Schuurmans Stekhoven $\mathrm{JH}$. Extent, distribution, and mammographic/histological correlation of breast ductal carcinoma in situ. Lancet 1990; 335: 519-22.

5. Henrot P, Leroux A, Barlier C, Genin P. Breast microcalcifications: The lesions in anatomical pathology. Diagn Interv Imaging 2014; 95: 141-52.

6. Faverly DR, Burgers L, Bult P, Holland R: Three dimensional imaging of mammary ductal carcinoma in situ: clinical implications. Semin Diagn Pathol 1994; 11: 193-8.

7. Mai KT, Yazdi HM, Burns BF, Perkins DG. Pattern of distribution of intraductal and infiltrating ductal carcinoma: a three-dimensional study using serial coronal giant sections of the breast. Hum Pathol 2000; 31: 464-74.

8. de Ross MAJ, Pijnappel RM, Post WJ, de Vries J, Baas PC, Groote LD. Correlation between imaging nad pathology in ductal carcinoma in situ of the breast. World J Surg Oncol 2004; 2: 4.
9. Lester SC, Bose S, Chen YY, Connolly JL, de Baca ME, Fitzgibbons PL, et al. Protocol for the examination of specimens from patients with ductal carcinoma in situ of the breast. Arch Pathol Lab Med 2009; 133: 15-25.

10. Schwartz GF, Lagios MD, Carter D, Connolly J, Ellis IO, Eusebi V, et al. Consensus conference on the classification of ductal carcinoma in situ. Cancer 1997; 80: 1798-802.

11. Tabar L, Tot T, Dean PB. Breast Cancer: The art and science of early detection with mammography. Stuttgart, New York: Thieme; 2005.

12. Silverstein MJ, Lagios MD. Treatment selection for patients with ductal carcinoma in situ (DCIS) of the breast using the University of Southern California/ Van Nuys (USC/VNPI) Prognostic Index. Breast J 2015; 21: 127-32.

13. Dinkel HP, Gassel AM, Tschammler A. Is the appearance of microcalcifications on mammography useful in predicting histological grade of malignancy in ductal cancer in situ? Br J Radiol 2000; 73: 938-44.

14. Slanetz PJ, Giardino AA, Oyama T, Koerner FC, Halpern EF, Moore RH, et al. Mammographic appearance of ductal carcinoma in situ does not reliably predict histologic subtype. Breast J 2001; 7: 417-21.

15. Holland R, Hendriks JH. Microcalcifications associated with ductal carcinoma in situ: mammographic-pathologic correlation. Semin Diagn Pathol 1994; 11: 181-92.

16. Evans A, Clements K, Maxwell A, Bishop H, Hanby A, Lawrence $G$, et al. Sloane Projest Steering Group: Lesion size is major determinant of the mammograhic features of ductal carcinoma in situ: findings from the Sloane Project. Clin Radiol 2010; 65: 181-4.

17. Barreau B, de Mascarel I, Feuga C, MacGrogan G, Dilhuydy MH, Picot V, et al. Mammography of ductal carcinoma in situ of the breast: review of 909 cases with radiographic-pathologic correlations. Eur J Radiol 2005; 54: 55-61.

18. Evans $A$, Pinder $S$, Wilson $R$, Sibbering $M$, et al. Ductal carcinoma in situ of the breast: correlation between mammographic and pathologic findings. AJR Am J Roentgenol 1994; 162: 1307-11.

19. Stomper PC, Connolly JL. Ductal carcinoma in situ of the breast: correlation between mammographic calcification and tumor subtype. AJR Am J Roentgenol 1992; 159: 483-5.

20. Tang X, Yamashita T, Hara M, Kumaki N, Tokuda Y, Masuda S. Histopathological characteristics of breast ductal carcinoma in situ and association with imaging findings. Breast Cancer 2015; Feb 3 [DOI10.1007/s12282-015-0592-0 Epub ahead of print].

21. Cho KR, Seo BK, Kim CH, Whang KW, Kim YH, Woo OH, et al. Non-calcified ductal carcinoma in situ: ultrasound and mammographic findings correlated with histological findings. Yonsei Med J 2008; 49: 103-10.

22. Schulz S, Sinn P, Golatta M, Rauch G, Junkermann H, Schuetz F, et al. Prediction of underestimated invasiveness in patients with ductal carcinoma in situ of the breast on percutaneous biopsy as rationale for recommending concurrent sentinel lymph node biopsy. Breast 2013; 22: 537-42.

23. Park HS, Park S, Cho J, Park JM, Kim SI, Park BW. Risk predictors of underestimation and the need for sentinel node biopsy in patients diagnosed with ductal carcinoma in situ by preoperative needle biopsy. J Surg Oncol 2013; 107: 388-92.

24. Szynglarewicz B, Kasprzak P, Halon A, Matkowski R. Preoperatively diagnosed ductal cancers in situ of the breast presenting as even small masses are of high risk for the invasive foci in postoperative specimen. World I Surg Oncol 2015; 13: 218.

25. Bae S, Yoon JH, Moon HJ, Kim MJ, Kim EK. Breast microcalcifications: diagnostic outcomes according to image-guided biopsy method. Korean J Radiol 2015; 16: 996-1005.

26. Rauch GM, Kuerer HM, Scoggins ME, Fox PS, Benveniste AP, Park YM, et al. Clinicopathologic, mammographic, and sonographic features in 1,187 patients with pure ductal carcinoma in situ of the breast by estrogen receptor status. Breast Cancer Res Treat 2013; 139: 639-47.

27. Mun HS, Shin HJ, Kim HH, Cha JH, Kim H. Screening-detected calcified and non-calcified ductal carcinoma in situ: differences in the imaging and histopathological features. Clin Radiol 2013; 68: e27-35.

28. Kim MY, Kim HS, Choi N, Yang JH, Yoo YB, Park KS. Screening mammographydetected ductal carcinoma in situ: mammographic features based on breast cancer subtypes. Clin Imaging 2015; 39: 983-6. 